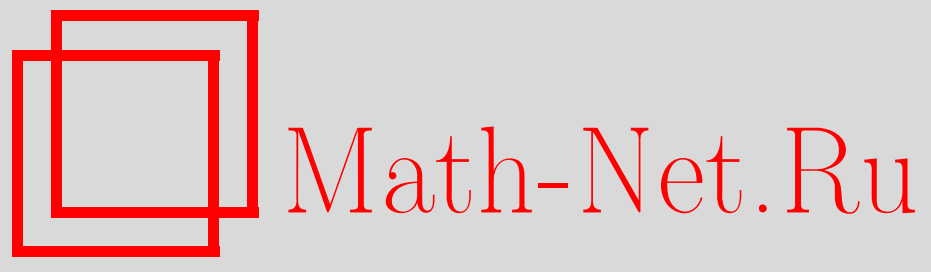

В. Д. Ляховский, Квантовая дуальность в квантовых деформациях, ТMФ, 2006, том 148, номер 1, 112-125

DOI: https://doi.org/10.4213/tmf2062

Использование Общероссийского математического портала Math-Net.Ru подразумевает, что вы прочитали и согласны с пользовательским соглашением http://www . mathnet.ru/rus/agreement

Параметры загрузки:

IP: 54.210 .77 .194

26 апреля 2023 г., 10:51:45

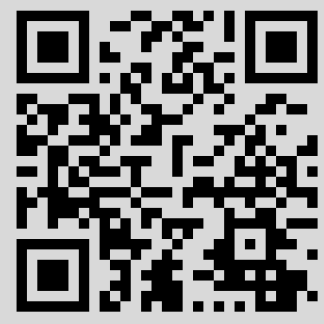




\title{
КВАНТОВАЯ ДУАЛЬНОСТЬ В КВАНТОВЫХ ДЕФОРМАЦИЯХ
}

\begin{abstract}
В соответствии с квантовым принципом дуальности скрученная алгебра $U_{\mathcal{F}}(\mathfrak{g})$ эквивалентна квантовой группе $\operatorname{Fun}_{\operatorname{def}}\left(\mathfrak{G}^{\#}\right)$ и имеет два предпочтительных базиса: первый наследуется из универсальной обертывающей алгебры $U(\mathfrak{g})$, второй порожден координатными функциями дуальной группы Ли $\mathfrak{G}^{\#}$. Продемонстрировано, как преобразование $\mathfrak{g} \longrightarrow \mathfrak{g}^{\#}$ может быть получено в явной форме для любой простой алгебры Ли и факторизуемой цепи $\mathcal{F}$ расширенных жордановых твистов. В алгебре $\mathfrak{g}^{\#}$ вводится естественная векторная градуировка $\Gamma\left(\mathfrak{g}^{\#}\right)$, согласованная с присоединенным представлением алгебры. Переход к координатам дуальной группы позволяет существенно упростить коструктуру деформированной алгебры Хопфа $U_{\mathcal{F}}(\mathfrak{g})$, рассматриваемой как квантовая группа $\operatorname{Fun}_{\text {def }}\left(\mathfrak{G}^{\#}\right)$. Преобразование $\mathfrak{g} \longrightarrow \mathfrak{g}^{\#}$ может быть использовано при построении новых решений уравнений твиста. Параметризованное семейство расширенных жордановых деформаций $U_{\mathcal{E} \mathcal{J}}(\mathfrak{s l}(3))$ конструируется и изучается в терминах $\mathcal{S} \mathcal{L}(3)^{\#}$, обнаружены новые реализации параболического твиста.
\end{abstract}

Ключевые слова: структуры Ли-Пуассона, квантовые деформации симметрии, квантовая дуальность.

Посвящается юбилею моего учителя

Юрия Викторовича Новожилова

\section{1. ВВЕДЕНИЕ}

В деформационном квантовании, где алгебра наблюдаемых деформируется "в направлении" скобки Пуассона и превращается в некоммутативную алгебру координатных функций, построение умножения в явном виде остается весьма сложной задачей [1]. Квантовый принцип дуальности [2], [3] позволяет строить деформированные группы с помощью квантовых универсальных обертывающих алгебр. Решающую роль в построении нестандартных квантовых алгебр для простых алгебр Ли играют постоянные треугольные решения уравнения Янга-Бакстера. Такие решения носят название скручиваний [4] и описывают структуры Ли-Пуассона, совместимые с алгебрами Ли $\mathfrak{g}$, т.е. механические системы, которые могут существовать на

${ }^{*}$ Санкт-Петербургский государственный университет, Санкт-Петербург, Россия. E-mail: lyakhovs@pobox.spbu.ru 
пространстве с фиксированной некоммутативностью. Скручивания $U(\mathfrak{g}) \longrightarrow U_{\mathcal{F}}(\mathfrak{g})$ обычно описываются в базисе Пуанкаре-Биркхофа-Витта (g-базисе) недеформированной алгебры Хопфа $U(\mathfrak{g})$ с умножением $m$ и коумножением $\Delta^{(0)}$, где $\Delta^{(0)}$ обозначает примитивное копроизведение: $\Delta^{(0)}(g)=g \otimes 1+1 \otimes g$ для $g \in \mathfrak{g}$. Применение $\mathfrak{g}$ базиса естественно, когда внимание сосредоточено на алгебраическом секторе алгебры Хопфа. Используя его, мы явно демонстрируем, что алгебраические соотношения в $U_{\mathcal{F}}(\mathfrak{g})$ остаются классическими, и умножение группы Ли-Пуассона зашифровано в элементе твиста $\mathcal{F}$. Результат скручивания посредством $\mathcal{F}: U(\mathfrak{g}) \longrightarrow U_{\mathcal{F}}(\mathfrak{g})$ [4] является алгеброй Хопфа $U_{\mathcal{F}}(\mathfrak{g})$ с теми же умножением $m$, единицей и коединицей, но с деформированными универсальным элементом $\mathcal{R}_{\mathcal{F}}=\mathcal{F}_{21} \mathcal{F}^{-1}$ и коумножением

$$
\Delta_{\mathcal{F}}=\mathcal{F} \Delta^{(0)} \mathcal{F}^{-1}
$$

Рассмотрим алгебру Хопфа $U_{\mathcal{F}}(\mathfrak{g})$ как квантованную биалгебру Ли $\left(\mathfrak{g}, \mathfrak{g}^{\#}\right)$, т.е. как деформацию $U(\mathfrak{g})$ в направлении $\mathfrak{g}^{\#}$. В соответствии с квантовым принципом дуальности алгебра $U_{\mathcal{F}}(\mathfrak{g})$, гладко скрученная твистом $\mathcal{F}$, является квантовой группой $\operatorname{Fun}_{\mathrm{def}}\left(\mathfrak{G}^{\#}\right)$ дуальной группы Ли $\mathfrak{G}^{\#}$. Группа $\mathfrak{G}^{\#}$ является универсальной накрывающей группой с алгеброй Ли $\mathfrak{g}^{\#}$. Для скрученной универсальной обертывающей алгебры $U_{\mathcal{F}}(\mathfrak{g})$ коалгебра Ли определяется классической $r$-матрицей,

$$
r=r^{l k} e_{l} \wedge e_{k}, \quad l, k=1, \ldots, m,
$$

и задается коскобкой

$$
\delta: \mathfrak{g} \longrightarrow \mathfrak{g} \otimes \mathfrak{g}, \quad \delta=\operatorname{ad}_{(r)} \circ \Delta^{(0)} .
$$

Минимальный набор $\left\{e_{l} \mid l=1, \ldots, m\right\}$, необходимый для задания $r$, образует базис подалгебры-носителя $\mathfrak{g}_{c}$ твиста $\mathcal{F}$. Коструктура $U_{\mathcal{F}}(\mathfrak{g})$ описывает закон композиции в $\operatorname{Fun}_{\mathrm{def}}\left(\mathfrak{G}^{\#}\right)$, т.е. деформированное умножение в $\operatorname{Fun}\left(\mathfrak{G}^{\#}\right)$. Деформация заключается в том, что координатные функции в $\operatorname{Fun}_{\mathrm{def}}\left(\mathfrak{G}^{\#}\right)$ не коммутируют, они подчинены закону композиции $m$ в $U(\mathfrak{g})$.

Структура дуальной алгебры Ли $\mathfrak{g}^{\#}$ может быть описана в терминах генераторов $\mathfrak{g}$ как "вторая" лиева структура на пространстве $\mathfrak{g}$. Когда алгебра $U(\mathfrak{g})$ деформируется с помощью твиста $\mathcal{F}$, это с очевидностью приводит не только к деформации (первоначально кокоммутативной) коструктуры, но также к преобразованию базиса в пространстве координатных функций. Это может быть продемонстрировано на примере жорданова твиста $\mathcal{F}_{\mathcal{J}}: U(\mathfrak{b}(2)) \longrightarrow U_{\mathcal{J}}(\mathfrak{b}(2))$ в $U(\mathfrak{b}(2))$. Носителем твиста $\mathcal{F}_{\mathcal{J}}=e^{H \otimes \sigma}$, где $\sigma=\ln (1+E)$, является алгебра $\mathfrak{b}(2)$ с генераторами $\{H, E\}$ и соотношением $[H, E]=E$. Дуальная алгебра $\mathfrak{b}(2) \#$ эквивалентна $\mathfrak{b}(2)$, поскольку классическая $r$-матрица $H \wedge E$ описывает изоморфизм

$$
r:\left\{\begin{aligned}
H^{*} & \Leftrightarrow E \\
E^{*} & \Leftrightarrow-H
\end{aligned}\right\} .
$$

Универсальный элемент для жорданова твиста $\mathcal{R}=\mathcal{F}_{21} \mathcal{F}^{-1}=e^{\sigma \otimes H} e^{-H \otimes \sigma}$ показывает, что в дуализации алгебр $U(\mathfrak{b}(2))$ и $U\left(\mathfrak{b}(2)^{\#}\right)$ генератор $E$ является лишь 
первым членом разложения $H^{*}$, в то время как элементом, дуальным к $H$, является формальный ряд $\sigma$. Тот же факт достаточно наглядно демонстрируется в коструктуре алгебры $U_{\mathcal{J}}(\mathfrak{b}(2))=\operatorname{Fun}_{\text {def }}(\mathfrak{B}(2) \#)$. Справедливы равенства

$$
\begin{aligned}
\Delta_{\mathcal{J}}(H) & =H \otimes e^{-\sigma}+1 \otimes H, \\
\Delta_{\mathcal{J}}(\sigma) & =\sigma \otimes 1+1 \otimes \sigma .
\end{aligned}
$$

Эти соотношения есть не что иное, как правила умножения в группе $\mathfrak{B}(2)^{\#}$ (полупрямом произведении "вращения" $\left(e^{-\sigma}\right)^{*}$ и “трансляции" $\left.H^{*}\right)$ с элементом Картана $\sigma$, который вместе с $H$ образует здесь $\mathfrak{g}^{\#-б а з и с ~-~ б а з и с ~ к о о р д и н а т н ы х ~ ф у н к ц и и ̆ ~ д у-~}$ альной группы.

Как мы показали выше, $\mathfrak{g}^{\#}$-координаты легко получить, если подалгебра-носитель твиста $\mathfrak{g}_{c}$ совпадает с $\mathfrak{g}$. В большинстве интересных случаев носителем является собственная подалгебра $\mathfrak{g}_{c} \subset \mathfrak{g}$. Подпространство $V_{\mathfrak{a}}$ в разложении $V_{\mathfrak{g}}=V_{\mathfrak{g}_{c}} \oplus V_{\mathfrak{a}}$ нетривиально, когда алгебра Ли $\mathfrak{g}$ полупроста [5]. На подпространстве $V_{\mathfrak{g}_{c}}$ и в алгебре Хопфа $U_{\mathcal{F}}\left(\mathfrak{g}_{c}\right)$ дуальные координаты $\mathfrak{g}^{\#}$ могут быть получены с помощью твиста $\mathcal{F}$ как отображение $\mathfrak{g} \longrightarrow \mathfrak{g}^{\#}$. Скручивание на пространстве $V_{\mathfrak{a}}$ обычно описывается набором копроизведений для базисных элементов в $\mathfrak{g}$ и выражается в терминах смешанного базиса, содержащего элементы как $\mathfrak{g}$-, так и $\mathfrak{g}_{c}^{\#}$-базисов. В такой реализации свойства дуальной группы оказываются зашифрованы в неадекватном базисе.

Деформированная коструктура является существенной не только сама по себе. Необходимо знать ее характеристики для того, чтобы найти дополнительные скручивания $U_{\mathcal{F}}(\mathfrak{g})$ и построить новые твисты, увеличивая число факторов в $\mathcal{F}$, другими словами, для того, чтобы найти факторизованные решения уравнений твиста [4]. В частности, особенно важными являются подпространства в $V_{\mathfrak{a}}$ с квазипримитивным коумножением [6], [7].

В этой работе мы рассмотрим универсальные обертывающие алгебры $U_{\mathcal{F}}(\mathfrak{g})$ для полупростых алгебр Ли $\mathfrak{g}$, скрученные цепями расширенных твистов - факторизуемыми 2-коциклами $\mathcal{F}=\mathcal{F}_{p} \mathcal{F}_{p-1} \ldots \mathcal{F}_{1}$, где каждый фактор $\mathcal{F}_{q}$ является решением уравнений твиста для $U_{\mathcal{F}_{q-1} \ldots \mathcal{F}_{1}}(\mathfrak{g})$. Эти решения (называемые твистовыми факторами [8]) бывают трех типов: жордановы факторы [9], факторы расширения [10] и абелевы факторы [11]. Абелев фактор в цепях $\mathcal{F}$ используется только для преобразований "вращения" жорданова скручивания и всегда может быть совмещен с соответствующим жордановым твистом.

В разделе 3 для дуальной алгебры $\mathfrak{g}^{\#}$ построена векторная градуировка, согласованная с ее присоединенным представленем. В разделе 4 разработан алгоритм для определения $\mathfrak{g}^{\#}$-координат в алгебре Хопфа $U_{\mathcal{F}}(\mathfrak{g})$. В разделе 5 реализация деформированной алгебры в базисе дуальной группы использована для изучения свойств алгебры Хопфа $U_{\mathcal{F}}(\mathfrak{s l}(3))$. Продемонстрировано, что дуальное представление коструктуры может быть использовано для поиска новых скручиваний. 


\section{2. ДУАЛЬНАЯ АЛГЕБРА ЛИ}

Рассмотрим простую алгебру Ли $\mathfrak{g}$ и базис Картана-Вейля $\left\{e_{i} \mid i=1, \ldots, n\right\}$, согласованный с разложением $V_{\mathfrak{g}}=V_{\mathfrak{h}} \oplus V_{\mathfrak{n}_{+}} \oplus V_{\mathfrak{n}_{-}}=V_{\mathfrak{h}} \bigoplus_{\nu \in \Lambda^{+}}\left(V_{\nu} \oplus V_{-\nu}\right)$,

$$
\begin{aligned}
V_{\mathfrak{n}_{+}} \oplus V_{\mathfrak{n}_{-}} & =\bigoplus_{\nu \in \Lambda^{+}}\left(V_{\nu} \oplus V_{-\nu}\right)= \\
& =V_{\lambda} \bigoplus_{\nu \in \Lambda^{+} \mid \nu\left(h_{\lambda}\right)=1 / 2}\left(V_{\nu} \oplus V_{-\nu}\right) \bigoplus_{\xi \in \Lambda^{+} \mid \xi\left(h_{\lambda}\right)=0}\left(V_{\xi} \oplus V_{-\lambda}\right)= \\
& =V_{1} \oplus V_{1 / 2} \oplus V_{0} \oplus V_{-1 / 2} \oplus V_{-1} .
\end{aligned}
$$

Здесь $\lambda$ - длинный корень и вектор $h_{\lambda}$ нормирован канонически: $\lambda\left(h_{\lambda}\right)=1$.

Подалгебра $\mathfrak{g}_{c}($ носитель цепи $\mathcal{F})$ является собственной в $\mathfrak{g}, \mathfrak{g}_{c} \subset \mathfrak{g}$. Генераторы $\left\{e_{l} \mid l=1, \ldots, m\right\}$ носителя $\mathfrak{g}_{c}$ содержат корневые векторы $\left\{e_{\phi} \mid \phi \in \Lambda_{c} \subset \Lambda(\mathfrak{g})\right\}$ для фиксированного набора корней $\Lambda_{c}[12]$. Пусть подпространство $h_{\left\{\lambda_{0}\right\}}^{\perp}$ ортогонально набору начальных корней $\lambda_{0}[6]$ в цепи $\mathcal{F}$. Рассмотрим подпространство $V_{\mathfrak{a}} \subset V_{\mathfrak{g}}$, порожденное элементами $\left\{e_{\nu} \mid \nu \in \Lambda \backslash \Lambda_{c}\right\}$ и подпространством $h_{\left\{\lambda_{0}\right\}}^{\perp}$ в подалгебpe Картана. Пространство алгебры есть прямая сумма: $V_{\mathfrak{g}}=V_{\mathfrak{g}_{c}} \oplus V_{\mathfrak{a}}$. Построим базис функционалов $\left\{e_{i}^{*}\right\}$, канонически дуальный к $\left\{e_{i}\right\}$, т.е. $e_{i}^{*}\left(e_{j}\right)=\delta_{i j}$. Пусть $\Gamma$ - весовая диаграмма присоединенного представления $\operatorname{ad}_{\mathfrak{g}}$. Наша цель - определить векторную градуировку $\Gamma^{\#}$ алгебры $\mathfrak{g}^{\#}$, согласованную с ее присоединенным действием. Структура Г\# описывается в следующем утверждении.

УТВЕРЖДЕНИЕ 1. Пусть $\mathfrak{g}$ - простая алгебра Ли с диаграммой весов присоединенного представления $\Gamma_{\mathrm{ad}(\mathfrak{g})}$ и разложением Картана $V_{\mathfrak{g}}=V_{\mathfrak{h}} \bigoplus_{\nu \in \Lambda^{+}}\left(V_{\nu} \oplus V_{-\nu}\right)$. $\Pi$ Путь

$$
\mathcal{F}=\mathcal{F}_{p} \mathcal{F}_{p-1} \ldots \mathcal{F}_{1}
$$

- решение уравнения скручивания, где каждый $\mathcal{F}_{q}$ является жордановым или расширенным твистовым фактором, и

$$
r_{\mathcal{F}}=\sum_{l, k=1, \ldots, \operatorname{dim}\left(\mathfrak{g}_{c}\right)} r^{l k} e_{l} \wedge e_{k} \in \mathfrak{g} \wedge \mathfrak{g}
$$

- соответствующая антисимметричная $r$-матрица, $\left(\mathfrak{g}, \mathfrak{g}^{\#}\right)$ - биалгебра Ли. Тогда векторная градуировка $\Gamma^{\#}$ дуальной алгебры Ли $\mathfrak{g}^{\#}$ является обгединением диаграммы $\Gamma_{c}^{\#}$, эквивалентной подсистеме $\Gamma_{\mathrm{ad}\left(\mathfrak{g}_{c}\right)}$ в $\Gamma_{\mathrm{ad}(\mathfrak{g})}$, и набора векторов $\Gamma_{a}^{\#}$, эквивалентного поддиаграмме в $\Gamma_{\mathrm{ad}(\mathfrak{g})}: \Gamma_{a}^{\#} \approx \Gamma_{\mathrm{ad}(\mathfrak{g})} \backslash \Gamma_{\mathrm{ad}\left(\mathfrak{g}_{c}\right)}$. Система $\Gamma_{c}^{\#}$ является набором весов $\left\{-\gamma_{i}\right\}$, противоположных весам носителя $\mathfrak{g}_{c}$. Для каждой компоненты $r^{\varphi \psi} \neq 0$ в $r$ генератор $\left(e_{\varphi}\right)^{*}$ получает вес $-\psi$, а генератор $\left(e_{\psi}\right)^{*}-$ вес $-\varphi$. Элементы $\left\{e_{\eta}^{*} \mid \eta \in \Gamma_{a}^{\#}\right\}$ генерируют абелев идеал $\mathfrak{g}_{a}^{\#}$ в дуалъной алгебре $\mathfrak{g}^{\#}$, которая является полупрямой суммой, $\mathfrak{g}^{\#} \approx \mathfrak{g}_{c}^{\#} \vdash \mathfrak{g}_{a}^{\#}$.

ДокАЗАТЕЛЬСтво. Рассмотрим композицию дуальной алгебры $\mathfrak{g}^{\#}$, записанную в виде коскобки $\delta(g)$ для $g \in \mathfrak{g}$ :

$$
\delta(g)=\left[r^{k l} e_{k} \wedge e_{l}, g \otimes 1+1 \otimes g\right]=r^{k l}\left[e_{k} g\right] \wedge e_{l}+r^{k l} e_{k} \wedge\left[e_{l} g\right] .
$$


Образ $\delta(g)$ отличен от нуля, если и только если один из его тензорных сомножителей принадлежит $\mathfrak{g}_{c}$. Если $g \in \mathfrak{g}_{c}$, то $\delta(g)$ принадлежит $\mathfrak{g}_{c} \otimes \mathfrak{g}_{c}$, поскольку $\mathfrak{g}_{c}$ является алгеброй. Тем самым доказано, что $\mathfrak{g}_{a}^{\#}$ является абелевым идеалом в $\mathfrak{g}^{\#}$.

Классическая $r$-матрица описывает деформацию (скручивание посредством $r$ ) биалгебры Ли, $r:(\mathfrak{g}, \mathrm{Ab}) \longrightarrow\left(\mathfrak{g}, \mathfrak{g}^{\#}\right)$ [2]. Каждое слагаемое в $r$ порождает деформирующую функцию для первоначального абелева закона композиции дуальной алгебры. Пусть $r$ - классическая $r$-матрица, соответствующая цепи расширенных твистов $\mathcal{F}(r)$. Чтобы найти структуру алгебры $\mathfrak{g}_{\mathcal{F}(r)}^{\#}$, достаточно рассмотреть деформирующие функции, происходящие из двух базисных твистовых факторов (БТФ): жорданова фактора и фактора расширения [10].

Жорданов БТФ имеет каноническую форму [9]:

$$
\widetilde{F}_{\mathrm{J}}=e^{h \otimes \sigma_{\lambda}}, \quad \sigma_{\lambda}=\ln \left(1+e_{\lambda}\right), \quad\left[h, e_{\lambda}\right]=e_{\lambda} .
$$

Он может быть деформирован предыдущими твистовыми факторами [7], [12] и приобрести более сложную структуру. Тем не менее во всех случаях его можно записать в виде

$$
\begin{gathered}
\widetilde{F}_{\mathrm{J}}=e^{h \otimes \tilde{\sigma}} \\
\tilde{\sigma}=\ln \left(1+f\left(e_{\lambda} ; e_{\alpha}, \xi_{q}\right)\right), \quad \lim _{\xi_{q} \rightarrow 0} f\left(e_{\lambda} ; e_{\alpha}, \xi_{q}\right)=e_{\lambda}
\end{gathered}
$$

$\left(\xi_{q}\right.$ являются деформирующими параметрами предшествующих скручиваний). Соответствующая жорданову БТФ $r$-матрица имеет вид

$$
r_{\mathrm{J}}=h \wedge e_{\lambda},
$$

где элемент Картана $h=h_{\lambda}+\gamma h_{\perp} \subset V_{\mathfrak{h}}, \quad \lambda\left(h_{\perp}\right)=0$, и $\left[h, e_{\lambda}\right]=\lambda(h) e_{\lambda} ; \quad \lambda(h)=$ $\lambda\left(h_{\lambda}\right)=1$. Элементы $h^{*}$ и $h_{\perp}^{*}$ канонически дуальны: $\left\langle h^{*}, h_{\perp}\right\rangle=0, \quad\left\langle h^{*}, h\right\rangle=$ $\left\langle h_{\perp}^{*}, h_{\perp}\right\rangle=1$. Коскобки на подалгебре $\mathfrak{g}_{\mathcal{J} c}=B(2)$ (носителе жорданова скручивания),

$$
\begin{aligned}
\delta(h) & =\left[h \otimes e_{\lambda}-e_{\lambda} \otimes h, h \otimes 1+1 \otimes h\right]=-h \wedge e_{\lambda}, \\
\delta\left(e_{\lambda}\right) & =\left[h \otimes e_{\lambda}-e_{\lambda} \otimes h, e_{\lambda} \otimes 1+1 \otimes e_{\lambda}\right]=0,
\end{aligned}
$$

показывают, что коммутационные соотношения в $\mathfrak{g}_{\mathcal{J} c}^{\#}$ определяются деформирующей функцией (гомоморфизмом пространств $\left.\mathfrak{g}^{\#} \wedge \mathfrak{g}^{\#} \longrightarrow \mathfrak{g}^{\#}[13]\right)$,

$$
f_{\mathrm{J}}^{\#}\left(e_{\lambda}^{*}, h^{*}\right)=h^{*},
$$

и $e_{\lambda}^{*}$ служат элементами Картана в $\mathfrak{g}_{\mathcal{J}}^{\#}$.

Рассмотрим $a_{\nu} \in V_{\mathfrak{a}}$. Имеем

$$
\delta\left(a_{\nu}\right)=\left[h \otimes e_{\lambda}-e_{\lambda} \otimes h, a_{\nu} \otimes 1+1 \otimes a_{\nu}\right]=\nu\left(h_{\lambda}+\gamma h_{\perp}\right) a_{\nu} \wedge e_{\lambda}+C_{\lambda \nu}^{\lambda+\nu} h \wedge a_{\lambda+\nu} .
$$

Отсюда следует, что

$$
\begin{gathered}
f_{\mathrm{J}}^{\#}\left(e_{\lambda}^{*}, a_{\nu}^{*}\right)=-\nu\left(h_{\lambda}+\gamma h_{\perp}\right) a_{\nu}^{*}, \\
f_{\mathrm{J}}^{\#}\left(h^{*}, a_{\lambda+\nu}^{*}\right)=C_{\lambda \nu}^{\lambda+\nu} a_{\nu}^{*},
\end{gathered}
$$


т.е. $h^{*}$ в базисе исходной корневой системы $\Lambda$ имеет градуировку $(-\lambda)$. Далее,

$$
\begin{gathered}
\delta\left(a_{-\lambda}\right)=\left[h \otimes e_{\lambda}-e_{\lambda} \otimes h, a_{-\lambda} \otimes 1+1 \otimes a_{-\lambda}\right]=e_{\lambda} \otimes a_{-\lambda}-2 \gamma h \wedge h_{\perp} ; \\
f_{\mathrm{J}}^{\#}\left(e_{\lambda}^{*}, a_{-\lambda}^{*}\right)=a_{-\lambda}^{*}, \quad f_{\mathrm{J}}^{\#}\left(h^{*}, h_{\perp}^{*}\right)=-2 \gamma a_{-\lambda}^{*} .
\end{gathered}
$$

Заметим, что ad" $\left(h^{*}\right)$ не "сдвигает" элемент $h_{\perp}^{*}$, если $h$ пропорционален $h_{\lambda}$. Для $h_{\perp}$ мы имеем $\delta\left(h_{\perp}\right)=0$.

Подводя итог, находим, что скручивание задается соотношениями

$$
\begin{array}{ll}
f_{\mathrm{J}}^{\#}\left(e_{\lambda}^{*}, h^{*}\right)=h^{*}, & f_{\mathrm{J}}^{\#}\left(h^{*}, a_{\lambda+\nu}^{*}\right)=C_{\lambda \nu}^{\lambda+\nu} a_{\nu}^{*}, \\
f_{\mathrm{J}}^{\#}\left(h^{*}, h_{\perp}^{*}\right)=-2 \gamma a_{-\lambda}^{*}, & f_{\mathrm{J}}^{\#}\left(e_{\lambda}^{*}, a_{-\lambda}^{*}\right)=a_{-\lambda}^{*}, \\
f_{\mathrm{J}}^{\#}\left(e_{\lambda}^{*}, a_{ \pm \nu}^{*}\right)=\mp \nu(h) a_{ \pm \nu}^{*} . &
\end{array}
$$

В этом случае деформирующая функция $f_{\mathrm{J}}^{\#}$ сама является композицией Ли, она фиксирует алгебру Ли $\mathfrak{g}^{\#}$, в которой $\mathfrak{g}_{\mathcal{J} c}^{\#}$ является подалгеброй, $\mathfrak{g}^{\#} \supset \mathfrak{g}_{\mathcal{J} c}^{\#}$. Ее присоединенное действие на $V_{\mathfrak{g}}^{*}$ может быть разложено в прямую сумму: $\left.\operatorname{ad}\left(\mathfrak{g}_{\mathcal{J} c}^{\#}\right)\right|_{V_{\mathfrak{g}}^{*}}=$ $\left.\left.\operatorname{ad}\left(\mathfrak{g}_{\mathcal{J} c}^{\#}\right)\right|_{V_{\mathfrak{g}_{\mathcal{J}}}^{*}} \oplus d\left(\mathfrak{g}_{\mathcal{J} c}^{\#}\right)\right|_{V_{\mathfrak{a}}^{*}}\left(\right.$ с представлением $d\left(\mathfrak{g}_{\mathcal{J} c}^{\#}\right)$, описывающим действие на $\left.V_{\mathfrak{a}}^{*}\right)$.

БТФ расширения. Рассмотрим элемент $r_{\mathrm{E}}=e_{\mu} \wedge e_{\nu}, \nu \neq-\mu$. Напомним, что сам элемент $r_{\mathrm{E}}$ не обязан удовлетворять классическому уравнению Янга-Бакстера, его решением должна быть только полная $r$-матрица. Построим коскобки

$$
\begin{aligned}
\delta(h) & =\left[e_{\mu} \otimes e_{\nu}-e_{\nu} \otimes e_{\mu}, h \otimes 1+1 \otimes h\right]=-(\mu+\nu)(h) e_{\mu} \wedge e_{\nu}, \\
\delta\left(e_{\xi}\right) & =\left[e_{\mu} \otimes e_{\nu}-e_{\nu} \otimes e_{\mu}, e_{\xi} \otimes 1+1 \otimes e_{\xi}\right]=C_{\mu \xi}^{\mu+\xi} e_{\mu+\xi} \wedge e_{\nu}+C_{\nu \xi}^{\nu+\xi} e_{\mu} \wedge e_{\nu+\xi}, \\
\delta\left(e_{-\mu}\right) & =\left[e_{\mu} \otimes e_{\nu}-e_{\nu} \otimes e_{\mu}, e_{-\mu} \otimes 1+1 \otimes e_{-\mu}\right]=2 h_{\mu} \wedge e_{\nu}+C_{\nu,-\mu}^{\nu-\mu} e_{\mu} \wedge e_{\nu-\mu},
\end{aligned}
$$

Если мы припишем базисному элементу $e_{\tau}^{*}$ вектор $\tau, \operatorname{to} \operatorname{ad}\left(e_{\mu}^{*}\right)$ и $\operatorname{ad}\left(e_{\nu}^{*}\right)$ будут действовать на $e_{\tau}^{*}$ как операторы, градуированные векторами $-\nu$ и $-\mu$, соответственно. Когда $h$ пропорционален $h_{\mu}$ (или $\left.h_{\nu}\right)$, оператор $\operatorname{ad}\left(e_{\nu}^{*}\right)\left(\right.$ или, соответственно, $\left.\operatorname{ad}\left(e_{\lambda}^{*}\right)\right)$ не может сдвинуть $h_{\perp}^{*}$.

Деформирующие функции $f_{\mathrm{E}}^{\#}$, соответствующие элементу $r_{\mathrm{E}}$, подразделяются на два набора. Первый набор описывает структуру дуального закона композиции на подпространстве $\mathfrak{g}_{c}^{\#}$ и свойства $\mathfrak{g}_{a}^{\#}$ как идеала:

$$
\begin{gathered}
f_{\mathrm{E}}^{\#}\left(e_{\mu+\xi}^{*}, e_{\nu}^{*}\right)=C_{\mu, \xi}^{\mu+\xi} e_{\xi}^{*}, \quad f_{\mathrm{E}}^{\#}\left(e_{\mu}^{*}, e_{\nu+\xi}^{*}\right)=C_{\nu, \xi}^{\nu+\xi} e_{\xi}^{*}, \quad \xi \neq \pm(\mu+\nu), \mu, \nu ; \\
f_{\mathrm{E}}^{\#}\left(e_{\mu}^{*}, e_{\nu-\mu}^{*}\right)=C_{\nu,-\mu}^{\nu-\mu} e_{-\mu}^{*}, \quad f_{\mathrm{E}}^{\#}\left(e_{\mu-\nu}^{*}, e_{\nu}^{*}\right)=C_{\mu,-\nu}^{\mu-\nu} e_{-\nu}^{*} ; \\
f_{\mathrm{E}}^{\#}\left(e_{\mu}^{*}, h_{\nu}^{*}\right)=2 e_{-\nu}^{*}, \quad f_{\mathrm{E}}^{\#}\left(h_{\mu}^{*}, e_{\nu}^{*}\right)=2 e_{-\mu}^{*} .
\end{gathered}
$$

Второй набор,

$$
\begin{gathered}
f_{\mathrm{E}}^{\#}\left(e_{\mu}^{*}, e_{\nu}^{*}\right)=-(\mu+\nu)(h) h^{*} \\
f_{\mathrm{E}}^{\#}\left(e_{\mu}^{*}, e_{\nu+\mu}^{*}\right)=C_{\nu, \mu}^{\nu+\mu} e_{\mu}^{*}, \quad f_{\mathrm{E}}^{\#}\left(e_{\nu+\mu}^{*}, e_{\nu}^{*}\right)=C_{\mu, \nu}^{\mu+\nu} e_{\nu}^{*},
\end{gathered}
$$


демонстрирует нарушения замкнутости композиции на пространстве $\mathfrak{g}_{c}^{\#}$. Указанные особенности характерны для элементов $r_{\mathrm{E}}$, которые не являются $r$-матрицей, т.е. соответствующие деформирующие функции сами по себе не задают закон композиции Ли.

Допустим, что сумма элементов $r_{\mathrm{J}}$ и $r_{\mathrm{E}}$ является $r$-матрицей (и $\lambda$ - длинный корень в $\Lambda)$. Это, в частности, означает, что $\left[e_{\mu}, e_{\nu}\right]=e_{\lambda}$. Поскольку нас интересуют скручивания посредством цепей $\mathcal{F}$ вида (4), то $\lambda$ - длинный корень и справедливо разложение (3). Соотношения (10), (11) становятся внутренними для $\mathfrak{g}^{\#}$. Деформирующие функции (9) должны быть объединены с (6). Элементы Картана в соответствующих коскобках становятся равными $\left(2 h_{\mu}-h\right)$ и $\left(2 h_{\nu}-h\right)$. В простой алгебре $\mathfrak{g}$ обе эти комбинации принадлежат пространству $h_{\left\{\lambda_{0}\right\}}^{\perp}$ (это свойство является прямым следствием возможности разложить систему положительных корней на старший корень, его “составляющие" и подсистему, ортогональную к старшему корню, подробности см. в [12]). В суммарной композиции [, ]\# присоединенные операторы $\operatorname{ad}\left(e_{\mu}^{\#}\right)$ и $\operatorname{ad}\left(e_{\nu}^{\#}\right)$ описывают действие подалгебры $\mathfrak{g}_{c}^{\#}$ на идеале $\mathfrak{g}_{a}^{\#}$.

Подведем итог. Объединяя свойства деформирующих функций для различных скручивающих факторов в $\mathcal{F}$, можсно убедиться, что в иелом для иепи расширенных твистов дуальный носитель $\mathfrak{g}_{c}^{\#}$ с лиевым умножением $f^{\#}=\sum f_{q}^{\#}$ является подалгеброй в $\mathfrak{g}^{\#}$, пространство дуальной алгебры эквивалентно прямой сумме $V_{\mathfrak{g}}^{*}=V_{\mathfrak{g}_{c}}^{*} \oplus V_{\mathfrak{a}}^{*}$, в то время как сама $\mathfrak{g}^{\#}$ является полупрямой суммой, $\mathfrak{g}^{\#} \approx \mathfrak{g}_{c}^{\#} \vdash \mathfrak{g}_{a}^{\#}$, с абелевым идеалом $\mathfrak{g}_{a}^{\#}$.

\section{3. ВТОРОЙ КЛАССИЧЕСКИЙ ПРЕДЕЛ ДЛЯ СКРУЧЕННЫХ АЛГЕБР}

В скрученной универсальной обертывающей алгебре, рассматриваемой как деформированная алгебра функций $U_{\mathcal{F}}(\mathfrak{g})=\operatorname{Fun}_{\mathrm{def}}\left(\mathfrak{G}^{\#}\right)$, группа $\mathfrak{G}^{\#}$ является универсальной накрывающей группой с алгеброй Ли $\mathfrak{g}^{\#}$ и может быть реализована в терминах формальных степенных рядов.

В предыдущем разделе было показано, как соотношения дуальной алгебры $\mathfrak{g}^{\#}$ описываются векторной диаграммой $\Gamma^{\#}$. Это описание относится к базису функционалов $e_{i}^{*}$, канонически дуальных к генераторам $\left\{e_{i}\right\}$ алгебры g. Таким образом, мы можем сконструировать групповое умножение для $\mathfrak{G}^{\#}$ в виде копроизведения в алгебре Хопфа $\operatorname{Fun}\left(\mathfrak{G}^{\#}\right)$ для элементов $e_{i}$, рассматриваемых как экспоненциальные координаты этой группы. Лишь в немногих тривиальных случаях преобразование к дуальным (некоммутативным) координатам может быть определено прямым сравнением $\operatorname{Fun}\left(\mathfrak{G}^{\#}\right)$ с $\operatorname{Fun}_{\text {def }}\left(\mathfrak{G}^{\#}\right)=U_{\mathcal{F}}(\mathfrak{g})$.

B алгебре Хопфа Fun $\operatorname{def}\left(\mathfrak{G}^{\#}\right)$ закон композиции группы $\mathfrak{G}^{\#}$ нарушен из-за некоммутативности координат. Обычно структурные константы квантовых деформаций строятся в терминах недеформированных координат. Другими словами, соответствующие координаты недеформированной группы Fun $\left(\mathfrak{G}^{\#}\right)$ должны быть достаточны для описания умножения деформированной группы $\operatorname{Fun}_{\mathrm{def}}\left(\mathfrak{G}^{\#}\right)$. Следовательно, для построения координат в интересующей нас алгебре Хопфа мы должны найти вспомогательную алгебру функций $\operatorname{Fun}\left(\mathfrak{G}^{\#}\right)$ с коммутативным умножением. 
Переход $\operatorname{Fun}_{\mathrm{def}}\left(\mathfrak{G}^{\#}\right) \longrightarrow \operatorname{Fun}\left(\mathfrak{G}^{\#}\right)$ называется вторым классическим пределом [2]. Было доказано [14], что для стандартного квантования (с параметром $q=e^{h}$ ) второй классический предел может быть получен с помощью тривиального скейлинга генераторов алгебры Ли. При этом вводится параметр скейлинга $\varepsilon$, и оба параметра $\varepsilon$ и $h$ устремляются к пределу $\varepsilon, h \longrightarrow 0$ при условии $h / \varepsilon=$ const. Принимая во внимание, что переход ко второму классическому пределу является общей процедурой, не зависящей от особенностей квантования, приходим к заключению, что это же свойство может быть использовано в скрученных универсальных обертывающих алгебрах $U_{\mathcal{F}}$.

Рассмотрим факторизованные твисты $\mathcal{F}=\mathcal{F}_{p} \mathcal{F}_{p-1} \ldots \mathcal{F}_{1}$ с жордановыми и расширяющими факторами $\mathcal{F}_{j}$. Последовательности $\mathcal{F}$ можно приписать набор параметров деформации $\left\{\xi_{s=1, \ldots, l} \mid l \leqslant p\right\}$, где $p$ равно числу независимых жордановых факторов $\mathcal{F}_{\mathcal{J} s}$. Следовательно, существуют параметризованное решение $r\left(\left\{\xi_{s}\right\}\right)$ классического уравнения Янга-Бакстера и параметризованные семейства алгебр $\mathfrak{g}^{\#}\left(\left\{\xi_{s}\right\}\right)$ и групп $\mathfrak{G}^{\#}\left(\left\{\xi_{s}\right\}\right)$.

Автоморфизм, который вводит параметры деформации вместе с указанным выше скейлингом, соответствует замене

$$
e_{j} \longrightarrow \frac{\xi_{s}^{\vartheta_{(s) j}}}{\varepsilon} \hat{e}_{j}
$$

(здесь степень $\vartheta_{(s)}$ является естественной градуировкой, соответствующей $s$-му звену цепи $\mathcal{F}$ ). Алгоритм классического предела состоит в том, что параметры $\varepsilon$ и $\xi_{s}$ должны стремиться к нулю, в то время как их отношения фиксируются:

$$
\varepsilon, \xi_{s} \longrightarrow 0, \quad \frac{\xi_{s}}{\varepsilon}=\zeta_{s} .
$$

В пределе имеем алгебру функций на недеформированной группе $\mathfrak{G}^{\#}\left(\left\{\zeta_{s}\right\}\right)$ :

$$
\lim _{\substack{\varepsilon, \xi_{s} \longrightarrow 0 ; \\ \xi_{s} / \varepsilon=\zeta_{s}}} U_{\mathcal{F}\left(\left\{\xi_{s}\right\}\right)}(\mathfrak{g}(\varepsilon))=\operatorname{Fun}\left(\mathfrak{G}^{\#}\left(\left\{\zeta_{s}\right\}\right)\right) .
$$

Нетрудно убедиться, что для канонически параметризованной цепи расширенных твистов $\mathcal{F}$ в пределе $(14)$ коструктурные константы $U_{\mathcal{F}\left(\left\{\xi_{s}\right\}\right)}(\mathfrak{g}(\varepsilon))$ остаются конечными, закон умножения становится абелевым, и алгебра Ли полученной группы совпадает с $\mathfrak{g}^{\#}\left(\left\{\zeta_{s}\right\}\right)$.

\section{4. ГРУППА Fun $_{\text {def }}\left(\mathfrak{G}^{\#}\right)$ В КООРДИНАТАХ ДУАЛЬНОЙ АЛГЕБРЫ}

В случае цепи $\mathcal{F}=\mathcal{F}_{p} \mathcal{F}_{p-1} \ldots \mathcal{F}_{1}$ дуальная группа $\mathfrak{G}^{\#}$ является разрешимой. Следовательно, экспоненциальные координаты могут рассматриваться как наиболее подходящие для описания $\operatorname{Fun}_{\operatorname{def}}\left(\mathfrak{G}^{\#}\right)=U_{\mathcal{F}}(\mathfrak{g})$. В этих координатах алгебра Хопфа $\left(\operatorname{Fun}_{\operatorname{def}}\left(\mathfrak{G}^{\#}\right)\right)^{*}$, дуальная к $\operatorname{Fun}_{\operatorname{def}}\left(\mathfrak{G}^{\#}\right)$ и являющаяся универсальной обертывающей алгеброй $U_{\text {def }}\left(\mathfrak{g}^{\#}\right)=\left(\operatorname{Fun}_{\operatorname{def}}\left(\mathfrak{G}^{\#}\right)\right)^{*}$, задана в базисе генераторов алгебры $\mathfrak{g}^{\#}$.

Алгебра $\mathfrak{g}^{\#}$ известна. Построив универсальную обертывающую алгебру $U\left(\mathfrak{g}^{\#}\right)$ в стандартном $\mathfrak{g}$-базисе, получим базис, дуальный к экспоненциальному координатному базису группы $\mathfrak{G}^{\#}$. Чтобы произвести преобразование координат, необходимо 
отождествить элементы алгебры Хопфа $U\left(\mathfrak{g}^{\#}\right)$ с элементами алгебры $\left(\operatorname{Fun}_{\mathrm{def}}\left(\mathfrak{G}^{\#}\right)\right)^{*}$. Последняя задана в терминах функционалов, канонически дуальных к элементам $\mathfrak{g}$-базиса в $U(\mathfrak{g})$. Иными словами, необходимо сравнить идеалы $I\left(\mathfrak{g}^{\#}\right)$ и $I\left(\mathfrak{g}_{(\mathfrak{g})}^{\#}\right)$, описывающие соотношения эквивалентности в соответствующих тензорных алгебрах. Это сопоставление приведет к набору равенств вида

$$
e_{i}^{\# *}=\phi_{i}^{*}\left(\left\{e_{j}^{*}\right\}\right),
$$

описывающих элементы $\mathfrak{g}^{\#}$-базиса в терминах $\mathfrak{g}$-базиса, и таким образом определяющих $\mathfrak{g}^{\#-к о о р д и н а т ы ~ б а з и с а ~ П у а н к а р е-Б и р к х о ф а-В и т т а ~ в ~}(\operatorname{Fun}(\mathfrak{G} \#))^{*}$. Переходя к соотношениям, дуальным к $(15)$, мы возвращаемся к $\operatorname{Fun}\left(\mathfrak{G}^{\#}\right)$ и находим искомое преобразование

$$
e_{i}^{\#}=\phi_{i}\left(\left\{e_{j}\right\}\right)
$$

Применив преобразование $\phi$ к деформированной алгебре $U_{\mathcal{F}}(\mathfrak{g})=\operatorname{Fun}_{\mathrm{def}}(\mathfrak{G} \#)$, получим умножение деформированной группы $\mathfrak{G}^{\#}$ в ее экспоненциальных координатах.

\section{5. ПРИМЕР. АЛГЕБРА $U_{\mathcal{F}}(s l(3))$}

\section{В КООРДИНАТАХ ДУАЛЬНОЙ ГРУППЫ $\mathcal{S L}(3)^{\#}$}

Пусть $\mathfrak{g}=\mathfrak{s l}(3)$. Рассмотрим компоненты $r$-матрицы $r_{\mathrm{J}}(\gamma)=h(\gamma) \wedge e_{13}$ и $r_{\mathrm{E}}=$ $e_{12} \wedge e_{23}$, где $h(\gamma)=h_{13}+\gamma h_{\perp}$. Сумма $r_{\mathrm{EJ}}(\gamma)=r_{\mathrm{J}}(\gamma)+r_{\mathrm{E}}$ является решением классического уравнения Янга-Бакстера. Последнее эквивалентно утверждению, что сумма деформирующих функций $f_{\mathrm{J}}^{\#}(\gamma)+f_{\mathrm{E}}^{\#}$ определяет умножение Ли [, ]\# := $\left(f_{\mathrm{J}}^{\#}(\gamma)+f_{\mathrm{E}}^{\#}\right)($,$) . На пространстве V_{\mathfrak{g}}^{*}$ этот закон умножения определяет дуальную алгебру $\mathfrak{s l}(3)^{\#}$ с соотношениями, легко вычисляемыми с помощью коскобки (5):

$$
\begin{gathered}
{\left[e_{13}^{*}, h^{*}(\gamma)\right]^{\#}=h^{*}(\gamma), \quad\left[h^{*}(\gamma), e_{12}^{*}\right]^{\#}=\left[h^{*}(\gamma), e_{23}^{*}\right]^{\#}=0,} \\
{\left[e_{13}^{*}, e_{21}^{*}\right]^{\#}=+\frac{1}{2}(3 \gamma+1) e_{21}^{*}, \quad\left[h^{*}(\gamma), h_{\perp}^{*}\right]^{\#}=-2 \gamma e_{31}^{*},} \\
{\left[e_{13}^{*}, e_{12}^{*}\right]^{\#}=-\frac{1}{2}(3 \gamma-1) e_{12}^{*}, \quad\left[e_{13}^{*}, e_{32}^{*}\right]^{\#}=-\frac{1}{2}(3 \gamma-1) e_{32}^{*},} \\
{\left[e_{13}^{*}, e_{31}^{*}\right]^{\#}=\left[e_{23}^{*}, e_{32}^{*}\right]^{\#}=\left[e_{12}^{*}, e_{21}^{*}\right]^{\#}=e_{31}^{*},} \\
{\left[h_{\perp}^{*}, e_{23}^{*}\right]^{\#}=(1-\gamma) e_{21}^{*}, \quad\left[h_{\perp}^{*}, e_{12}^{*}\right]^{\#}=(1+\gamma) e_{32}^{*},} \\
{\left[e_{13}^{*}, e_{23}^{*}\right]^{\#}=+\frac{1}{2}(3 \gamma+1) e_{23}^{*}, \quad\left[e_{12}^{*}, e_{23}^{*}\right]^{\#}=-h^{*}(\gamma) .}
\end{gathered}
$$

Эта алгебра имеет четырехмерную подалгебру (эквивалентную подалгебре $\mathfrak{g}_{c}-$ носителю $r$-матрицы $\left.r_{\mathrm{EJ}}(\gamma)\right)$ с векторной диаграммой $\Gamma_{c}^{\#}=\left\{0, \varphi_{31}, \varphi_{32}, \varphi_{21}\right\}$ и четырехмерный абелев идеал, генерированный набором $\left\{h_{\perp}^{*}, e_{31}^{*}, e_{32}^{*}, e_{21}^{*}\right\}$ с диаграммой $\Gamma_{a}^{\#}=\left\{0, \rho_{31}, \rho_{32}, \rho_{21}\right\}$. Здесь $\left\{\varphi_{a b}\right\}$ и $\left\{\rho_{a b}\right\}-$ копии тех векторов из $\Lambda(\mathfrak{s l}(3))$, которые соответствуют генераторам $e_{a b}$. В рассматриваемом случае обе подсистемы содержат одни и те же наборы векторов. Действие $\operatorname{ad}\left(\mathfrak{g}_{c}^{\#}\right)$ на $h_{\perp}^{*}$ показывает, что функционал $h_{\perp}^{*}$ ведет себя как “ортогональный” к $h^{*}$. 
Как продемонстрировано в [10], выражение

$$
\mathcal{F}(\gamma)=\mathcal{F}_{\mathrm{E}}(\gamma) \mathcal{F}_{\mathrm{J}}(\gamma)=\exp \left(\xi e_{12} \otimes e_{23} e^{(3 \gamma-1) \sigma(\xi) / 2}\right) \exp (h \otimes \sigma(\xi))
$$

является решением уравнений скручивания, соответствующим $r$-матрице $r_{\mathrm{EJ}}(\gamma)$. Здесь

$$
\sigma(\xi)=\ln \left(1+\xi e_{13}\right), \quad h=h_{13}+\gamma h_{\perp}, \quad h_{\perp}=\frac{1}{2} e_{11}-e_{22}+\frac{1}{2} e_{33} .
$$

Параметр $\gamma$ относится к скручиванию Решетихина [11], которое в данном случае объединено с жордановым фактором, $\xi$ является параметром деформации, соответствующим автоморфизму $e_{\tau} \longrightarrow \xi^{\tau\left(h_{13}\right)} \tilde{e}_{\tau}$. Введя скейлинг, получим параметризацию, достаточную для установления второго классического предела

$$
e_{\tau} \longrightarrow \frac{\xi^{\tau\left(h_{13}\right)}}{\varepsilon} \hat{e}_{\tau}, \quad \frac{\xi}{\varepsilon}=\zeta
$$

Произведем скручивание посредством $\mathcal{F}(\xi, \varepsilon, \zeta)$, совершим предельный переход (13) и получим умножение в дуальной группе $\mathfrak{G}^{\#}=(\mathfrak{S} \mathfrak{L}(3))^{\#}$ в $\mathfrak{s l}(3)$-координатах $\left\{\widehat{e_{j}}, \hat{\sigma}, \hat{h}, \hat{h}_{\perp}\right\}$ :

$$
\begin{aligned}
& \Delta_{\mathcal{F}} \lim _{h}(\hat{h}(\gamma))=\hat{h}(\gamma) \otimes e^{-\hat{\sigma}(\zeta)}+1 \otimes \hat{h}(\gamma)-\xi \hat{e}_{12} \otimes \hat{e}_{23} e^{(\gamma-1) \hat{\sigma}(\zeta) / 2} ; \\
& \Delta_{\mathcal{F}}^{\lim }\left(\hat{h}_{\perp}\right)=\hat{h}_{\perp} \otimes 1+1 \otimes \hat{h}_{\perp} ; \\
& \Delta_{\mathcal{F}}^{\lim }\left(\hat{e}_{12}\right)=\hat{e}_{12} \otimes e^{(3 \gamma-1) \hat{\sigma}(\zeta) / 2}+1 \otimes \hat{e}_{12} ; \\
& \Delta_{\mathcal{F}}^{\lim }\left(\hat{e}_{23}\right)=\hat{e}_{23} \otimes e^{-(3 \gamma-1) \hat{\sigma}(\zeta) / 2}+e^{\hat{\sigma}(\zeta)} \otimes \widehat{e_{23}} ; \\
& \Delta_{\mathcal{F}}(\hat{\sigma}(\zeta))=\hat{\sigma}(\zeta) \otimes 1+1 \otimes \hat{\sigma}(\zeta) ; \\
& \Delta_{\mathcal{F}}^{\lim }\left(\hat{e}_{21}\right)=\hat{e}_{21} \otimes e^{-(3 \gamma+1) \hat{\sigma}(\zeta) / 2}+1 \otimes \hat{e}_{21}+\zeta(1-\gamma) \hat{h}_{\perp} \otimes \hat{e}_{23} e^{-\hat{\sigma}(\zeta)} ; \\
& \Delta_{\mathcal{F}}^{\lim }\left(\hat{e}_{32}\right)=\hat{e}_{32} \otimes e^{(3 \gamma-1) \hat{\sigma}(\zeta) / 2}+1 \otimes \hat{e}_{32}+\zeta \hat{h}(\gamma) \otimes \hat{e}_{12} e^{-\hat{\sigma}(\zeta)}+ \\
& +\zeta \hat{e}_{12} \otimes\left(\hat{h}(\gamma)-(\gamma+1) \widehat{h_{\perp}}\right) e^{(3 \gamma-1) \hat{\sigma}(\zeta) / 2}- \\
& -\zeta \hat{h}(\gamma) \hat{e}_{12} \otimes\left(e^{(3 \gamma-1) \hat{\sigma}(\zeta) / 2}-e^{3(\gamma-1) \hat{\sigma}(\zeta) / 2}\right)- \\
& -\zeta^{2} \hat{e}_{12} \otimes \hat{e}_{23} \hat{e}_{12} e^{3(\gamma-1) \hat{\sigma}(\zeta) / 2}-\zeta^{2} \hat{e}_{12}^{2} \otimes \hat{e}_{23} e^{(3 \gamma-2) \hat{\sigma}(\zeta)} ; \\
& \left.\Delta_{\mathcal{F}} \lim _{\hat{e}_{31}}\right)=\hat{e}_{31} \otimes e^{-\hat{\sigma}(\zeta)}+1 \otimes \hat{e}_{31}+ \\
& +2 \zeta \hat{h}(\gamma) \otimes\left(\hat{h}(\gamma)-\gamma \hat{h}_{\perp}\right) e^{-\hat{\sigma}(\zeta)}- \\
& -\zeta \hat{h}(\gamma)\left(\hat{h}(\gamma)-2 \gamma \hat{h}_{\perp}\right) \otimes\left(e^{-\hat{\sigma}(\zeta)}-e^{-2 \hat{\sigma}(\zeta)}\right)+ \\
& +\zeta^{2} \hat{h}(\gamma) \hat{e}_{12} \otimes \hat{e}_{23}\left(e^{3(\gamma-1) \hat{\sigma}(\zeta) / 2}-2 e^{(3 \gamma-5) \hat{\sigma}(\zeta) / 2}\right)+ \\
& +\zeta \hat{e}_{12} \otimes \hat{e}_{21} e^{(3 \gamma-1) \hat{\sigma}(\zeta) / 2}-\zeta \hat{e}_{32} \otimes \hat{e}_{23} e^{3(\gamma-1) \hat{\sigma}(\zeta) / 2}- \\
& -2 \zeta^{2} \hat{e}_{12} \otimes\left(\hat{h}(\gamma)-\gamma \hat{h}_{\perp}\right) \hat{e}_{23} e^{3(\gamma-1) \hat{\sigma}(\zeta) / 2}+ \\
& +\zeta \hat{e}_{12}^{2} \otimes \hat{e}_{23}^{2} e^{3(\gamma-1) \hat{\sigma}(\zeta)} \text {. }
\end{aligned}
$$

Каноническая дуализация переводит эту коструктуру в умножение алгебры Хопфа $\left(U_{\mathcal{F}}(\mathfrak{s l}(3))\right)^{*}=\left(\operatorname{Fun}\left(\mathfrak{S} \mathfrak{L}(3)^{\#}\right)\right)^{*}=U\left((\mathfrak{s l}(3))_{\mathfrak{g}}^{\#}\right)$. Таким образом, оно оказывается 
реализованным в терминах $\mathfrak{g}$-координат $\left\{\hat{e}_{\tau}^{*}\right\}$. Та же алгебра Хопфа в терминах

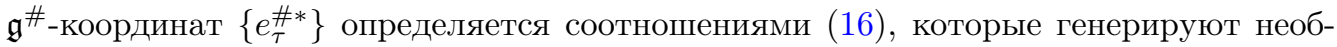
ходимый идеал в тензорной алгебре над $V_{\mathfrak{g}}$. Деформационный параметр $\zeta$ определяется скейлингом в (18). Сравнивая ассоциативные умножения в $U\left((\mathfrak{s l}(3))^{\#}\right)$ и $U\left((\mathfrak{s l}(3))_{\mathfrak{g}}^{\#}\right)$, находим два типа соотношений. Соотношениями первого типа являются

$$
\begin{aligned}
& \widehat{\sigma(\zeta)}^{*} \cdot \widehat{h(\gamma)}^{*}=\widehat{h(\gamma)}^{*}, \ldots ; \quad e_{13}^{\# *}(\zeta) \cdot h(\gamma)^{\# *}=h(\gamma)^{\# *}, \ldots ; \\
& \widehat{\sigma(\zeta)}^{*} \cdot\left(\hat{e}_{23} e^{-\widehat{\sigma(\zeta)}}\right)^{*}=\frac{1}{2}\left(\hat{e}_{23} e^{-\widehat{\sigma(\zeta)}}\right)^{*} ; \quad e_{13}^{\# *}(\zeta) \cdot e_{23}^{\# *}=\frac{1}{2} e_{23}^{\# *} \text {. }
\end{aligned}
$$

Они означают, что переход к $\mathfrak{g}^{\#}$-генераторам должен включать замены

$$
e_{13}^{\#}(\zeta)=\ln \left(1+\zeta \widehat{e_{13}}\right), \quad e_{23}^{\#}=\hat{e}_{23} e^{-\widehat{\sigma(\zeta)}} .
$$

Эти преобразования осуществляются в пространстве $U\left(\mathfrak{g}_{c}^{\#}\right)$ подалгебры носителя. Они совпадают с морфизмами, заданными твистом $\mathcal{F}: \mathfrak{g}_{c} \longrightarrow \mathfrak{g}_{c}^{\#}$.

Более важными являются соотношения другого типа на пространстве $V_{\mathfrak{a} \#}$, дополнительном к $V_{\mathfrak{g}_{c}^{\#}}$. Они не могут быть непосредственно генерированы скручивающим элементом и зависят от подпредставления $\left.\mathrm{ad} \mathfrak{g}_{c}^{\#}\right|_{V_{\mathfrak{a}}}$. В нашем случае существуют только по два соотношения этого типа, полученные из (19) и (16):

$$
\widehat{h(\gamma)}^{*} \cdot \hat{e}_{12}^{*}=\left(\widehat{h(\gamma)} \cdot \hat{e}_{12}\right)^{*}+\zeta \hat{e}_{12}^{*}, \quad \widehat{h(\gamma)}^{*} \cdot \widehat{h(\gamma)}^{*}=2\left((\widehat{h(\gamma)})^{2}\right)^{*}+2 \zeta \hat{e}_{31}^{*}
$$

И

$$
h(\gamma)^{\# *} \cdot e_{12}^{\# *}=\left(h(\gamma)^{\#} \cdot e_{12}^{\#}\right)^{*}, \quad h(\gamma)^{\# *} \cdot h(\gamma)^{\# *}=2\left(\left(h(\gamma)^{\#}\right)^{2}\right)^{*},
$$

соответственно. Они приводят к двум нетривиальным соотношениям между $(\mathfrak{g})^{*}-$ и $\left(\mathfrak{g}^{\#}\right)^{*}$-генераторами:

$$
\begin{aligned}
\left(\widehat{h(\gamma)} \cdot \hat{e}_{12}\right)^{*}(\zeta) & =\left(h(\gamma)^{\#} \cdot e_{12}^{\#}\right)^{*}-\zeta e_{32}^{\# *} \\
\left((\widehat{h(\gamma)})^{2}\right)^{*}(\zeta) & =\frac{1}{2}\left(\left(h(\gamma)^{\#}\right)^{2}\right)^{*}-\zeta e_{31}^{\# *}
\end{aligned}
$$

и к соответствующим соотношениям между $\mathfrak{g}$ - и $\mathfrak{g}^{\#}$-генераторами:

$$
\begin{aligned}
& e_{32}^{\#}(\zeta)=\hat{e}_{32}-\zeta \widehat{h(\gamma)} \hat{e}_{12}, \\
& e_{31}^{\#}(\zeta)=\hat{e}_{31}-\zeta(\widehat{h(\gamma)})^{2} .
\end{aligned}
$$

Как уже упоминалось выше, элементы $\left\{e_{\tau}^{\#}\right\}$, построенные в терминах координат $\hat{e}_{\tau}$ недеформированной группы, подходят также для описания скрученной алгебры Хопфа. В итоге мы имеем следующее преобразование:

$$
\begin{array}{ll}
e_{13}^{\#}(\zeta)=\sigma(\zeta), & e_{23}^{\#}(\zeta)=e_{23} e^{-\sigma(\zeta)}, \\
e_{32}^{\#}(\zeta)=e_{32}-\zeta h(\gamma) e_{12}, & e_{31}^{\#}(\zeta)=e_{31}-\zeta h(\gamma)^{2} .
\end{array}
$$


В терминах $\mathfrak{g}$-координат (и функции $\left.\sigma(\xi)=\ln \left(1+\xi e_{13}\right)\right)$ коструктура скрученной алгебры $U_{\mathcal{F}}(\mathfrak{s l}(3))$ на подпространстве $V_{\mathfrak{a}}^{\#}$ определяется соотношениями [10], [15]:

$$
\begin{aligned}
\Delta_{\mathcal{F}}\left(h_{\perp}\right)= & h_{\perp} \otimes 1+1 \otimes h_{\perp} \\
\Delta_{\mathcal{F}}\left(e_{21}\right)= & e_{21} \otimes e^{-(3 \gamma+1) \sigma(\xi) / 2}+1 \otimes e_{21}+\xi(1-\gamma) h_{\perp} \otimes e_{23} e^{-\sigma(\xi)} \\
\Delta_{\mathcal{F}}\left(e_{32}\right)= & e_{32} \otimes e^{(3 \gamma-1) \sigma(\xi) / 2}+1 \otimes e_{32}+\xi h(\gamma) \otimes e_{12} e^{-\sigma(\xi)}+ \\
& +\xi e_{12} \otimes\left(h(\gamma)-(\gamma+1) h_{\perp}\right) e^{(3 \gamma-1) \sigma(\xi) / 2}- \\
& -\xi h(\gamma) e_{12} \otimes\left(e^{(3 \gamma-1) \sigma(\xi) / 2}-e^{(\gamma-1) \sigma(\xi) / 2}\right)- \\
& -\xi^{2} e_{12} \otimes e_{23} e_{12} e^{3(\gamma-1) \sigma(\xi) / 2}-\xi^{2} e_{12}^{2} \otimes e_{23} e^{(3 \gamma-2) \sigma(\xi)} \\
\Delta_{\mathcal{F}}\left(e_{31}\right)=e_{31} \otimes & e^{-\sigma(\xi)}+1 \otimes e_{31}+ \\
+ & 2 \xi h(\gamma) \otimes\left(h(\gamma)-\gamma h_{\perp}\right) e^{-\sigma(\xi)}+ \\
+ & \xi\left(h(\gamma)-h(\gamma)^{2}\right) \otimes\left(e^{-\sigma(\xi)}-e^{-2 \sigma(\xi)}\right)+ \\
+ & \xi e_{12} \otimes e_{21} e^{(3 \gamma-1) \sigma(\xi) / 2}- \\
- & \xi e_{32} \otimes e_{23} e^{3(\gamma-1) \sigma(\xi) / 2}+\xi^{2} h(\gamma) e_{12} \otimes e_{23} e^{3(\gamma-1) \sigma(\xi) / 2}+ \\
+ & 2 \xi^{2} e_{12} \otimes e_{23} e^{(3 \gamma-5) \sigma(\xi) / 2}-\xi^{2} e_{12} \otimes e_{23} e^{3(\gamma-1) \sigma(\xi) / 2}- \\
- & 2 \xi^{2} e_{12} \otimes\left(h(\gamma)-\gamma h_{\perp}\right) e_{23} e^{3(\gamma-1) \sigma(\xi) / 2}- \\
- & 2 \xi^{2} h(\gamma) e_{12} \otimes e_{23} e^{(3 \gamma-5) \sigma(\xi) / 2}+\xi^{3} e_{12}^{2} \otimes e_{23}^{2} e^{3(\gamma-1) \sigma(\xi)}
\end{aligned}
$$

Применяя к этим копроизведениям преобразование (20), мы получаем ту же коструктуру в $\mathfrak{g}^{\#}$-координатах:

$$
\begin{aligned}
\Delta_{\mathcal{F}}\left(h_{\perp}^{\#}\right)= & h_{\perp}^{\#} \otimes 1+1 \otimes h_{\perp}^{\#} ; \\
\Delta_{\mathcal{F}}\left(e_{21}^{\#}\right)= & e_{21}^{\#} \otimes e^{-((3 \gamma+1) / 2) e_{13}^{\#}}+1 \otimes e_{21}^{\#}+\zeta(1-\gamma) h_{\perp}^{\#} \otimes e_{23}^{\#} ; \\
\Delta_{\mathcal{F}}\left(e_{32}^{\#}\right)= & e_{32}^{\#} \otimes e^{((3 \gamma-1) / 2) e_{13}^{\#}}+1 \otimes e_{32}^{\#}-\zeta(\gamma+1) e_{12}^{\#} \otimes h_{\perp}^{\#} e^{((3 \gamma-1) / 2) e_{13}^{\#}} \\
\Delta_{\mathcal{F}}\left(e_{31}^{\#}\right)= & e_{31}^{\#} \otimes e^{-e_{13}^{\#}}+1 \otimes e_{31}^{\#}+ \\
& +\zeta e_{12}^{\#} \otimes e_{21}^{\#} e^{((3 \gamma-1) / 2) e_{13}^{\#}}-\zeta e_{32}^{\#} \otimes e_{23}^{\#} e^{3((\gamma-1) / 2) e_{13}^{\#}}- \\
& -2 \zeta \gamma h^{\#}(\gamma) \otimes h_{\perp}^{\#} e^{-e_{13}^{\#}}+2 \zeta^{2} \gamma e_{12}^{\#} \otimes h_{\perp}^{\#} e_{23}^{\#} e^{3((\gamma-1) / 2) e_{13}^{\#}}
\end{aligned}
$$

Эта реализация наглядно демонстрирует присоединенное действие группы $\mathfrak{G}_{c}^{\#}$ на четырехмерном пространстве $V_{\mathfrak{a} \# \text {. }}$ В стандартном ортогональном базисе $\left\{\mathbf{e}_{i}\right\}$ векторы диаграммы

$$
\Gamma_{\mathcal{F}}^{\#}=\Gamma_{c}^{\#} \cup \Gamma_{a}^{\#}=\left\{0, \varphi_{31}, \varphi_{32}, \varphi_{21}\right\} \cup\left\{0, \rho_{31}, \rho_{32}, \rho_{21}\right\}
$$

имеют вид

$$
\varphi_{a b}=\rho_{a b}=\mathbf{e}_{a}-\mathbf{e}_{b} .
$$

Рассмотрим, например, копроизведение $\Delta_{\mathcal{F}}\left(e_{31}^{\#}\right)$. Первые два слагаемых появились благодаря тривиальному свойству единицы и коммутатора

$$
\left[e_{13}^{\# *}, e_{31}^{\# *}\right]=\cdots+e_{31}^{\# *}+\cdots .
$$


Остальные четыре члена связаны со сдвигами, порождающими вектор $\rho_{31}$ :

$$
\varphi_{32} \circ \rho_{21}=\varphi_{21} \circ \rho_{32}=\varphi_{31} \circ \rho\left(h_{\perp}^{\#}\right)=\varphi_{32} \circ \varphi_{21} \circ \rho\left(h_{\perp}^{\#}\right)=\rho_{31} .
$$

Преобразованная коструктура (23) позволяет сформулировать в явном виде свойства, необходимые для построения новых скручивающих коциклов. Прежде всего, становится очевидным, что параметризованный набор дуальных групп $\mathfrak{G} \#(\gamma)$ имеет три особые точки: $\gamma=0, \pm 1$. В точке $\gamma=0$ копроизведения для $e_{31}^{\#}$ не имеют членов, содержащих $h_{\perp}^{\#}$. Результаты сдвигов $\varphi_{31} \circ \rho\left(h_{\perp}^{\#}\right)$ и $\varphi_{32} \circ \varphi_{21} \circ \rho\left(h_{\perp}^{\#}\right)$ равны нулю, так как в этом случае $h^{\#}$ ортогонален весу $h_{\perp}^{\#}$. Точки $\gamma=+1 \quad(\gamma=-1)$ особенно интересны. В них копроизведение $\Delta_{\mathcal{F}}\left(e_{32}^{\#}\right)\left(\right.$ соответственно $\left.\Delta_{\mathcal{F}}\left(e_{21}^{\#}\right)\right)$ становится квазипримитивным. Вместе с примитивностью $\Delta_{\mathcal{F}}\left(h_{\perp}^{\#}\right)$ это означает, что в этих точках уравнения скручивания для алгебры $U_{\mathcal{F}}(\mathfrak{s l}(3))$ имеют дополнительные решения

$$
\begin{aligned}
\mathcal{F}_{\mathcal{J}_{P+}} & =\exp \left(-\frac{2}{3} h_{\perp} \otimes \ln \left(\left(1+e_{21}\right) e^{2 \sigma_{13}}\right)\right), \quad \gamma=+1 \\
\mathcal{F}_{\mathcal{J}_{P-}} & =\exp \left(+\frac{2}{3} h_{\perp}^{\#} \otimes \ln \left(\left(1+e_{32}^{\#}\right) e^{2 e_{13}^{\#}}\right)\right)= \\
& =\exp \left(+\frac{2}{3} h_{\perp} \otimes \ln \left(\left(1+e_{32}-\zeta h e_{12}\right) e^{2 \sigma_{13}(\zeta)}\right)\right), \quad \gamma=-1 .
\end{aligned}
$$

Соответственно для $U(\mathfrak{s l}(3))$ мы имеем два параболических твиста

$$
\begin{array}{ll}
\mathcal{F}_{\mathfrak{P}+}=\mathcal{F}_{\mathcal{J}_{P+}} \mathcal{F}_{\mathcal{E}} \mathcal{F}_{\mathcal{J}}, & \gamma=+1 \\
\mathcal{F}_{\mathfrak{P}-}=\mathcal{F}_{\mathcal{J}_{P-}} \mathcal{F}_{\mathcal{E}} \mathcal{F}_{\mathcal{J}}, & \gamma=-1 .
\end{array}
$$

Эта конструкция индуцирует параболические скручивания, первое из которых было впервые получено в [16]. Использование координат дуальной группы позволяет установить, что для алгебры $U(\mathfrak{s l}(3))$ имеется два неэквивалентных параболических скручивания. Они реализуют квантования параболических $r$-матриц:

$$
\begin{aligned}
& r_{\mathfrak{P}+}=\xi\left(e_{12} \wedge e_{23}+\left(h_{13}+h_{\perp}\right) \wedge e_{13}\right)-\frac{2}{3} h_{\perp} \wedge e_{21}, \\
& r_{\mathfrak{P}-}=\xi\left(e_{12} \wedge e_{23}+\left(h_{13}+h_{\perp}\right) \wedge e_{13}\right)+\frac{2}{3} h_{\perp} \wedge e_{32} .
\end{aligned}
$$

Твисты $\mathcal{F}_{\mathcal{J}_{P+}}$ и $\mathcal{F}_{\mathcal{J}_{P-}}$ являются деформированными жордановыми факторами.

\section{6. ЗАКЛЮЧЕНИЕ}

Мы продемонстировали, что координаты дуальной группы обеспечивают естественный базис для коструктуры скрученных универсальных обертывающих алгебр. В случае разрешимой группы $\mathfrak{G}^{\#}$ ее копроизведение приобретает в $\mathfrak{g}^{\#}$-координатах особенно простой вид, соответствующий экспоненциальному отображению присоединенного действия алгебры $\mathfrak{g} \#$. В скрученной алгебре $U_{\mathcal{F}}(\mathfrak{g})$ переход к координатам дуальной группы упрощает задачу построения новых твистов. 
Подход, основанный на квантовом принципе дуальности, позволяет по-новому интерпретировать построение пространств деформированных носителей [7], [12]. В диаграмме $\Gamma_{\mathcal{F}}^{\#}$ векторы $\lambda_{\perp}$, ортогональные начальному корню $\lambda$ полного расширенного твиста $\mathcal{F}$, не могут быть достигнуты из точек $\Gamma_{a}^{\#}$ с помощью сдвигов, соответствующих векторам в $\Gamma_{c}^{\#}$. Причина заключается в том, что диаграмма $\Gamma_{c}^{\#}$ находится в отрицательном секторе, в то время как $\Gamma_{\perp \lambda_{0}}$ лежит в нулевой плоскости, $\lambda_{\perp}\left(h_{\lambda_{0}}\right)=0$. Для канонических расширенных твистов (без "вращений") это означает, что копроизведения $\Delta_{\mathcal{F}}\left(e_{\lambda_{\perp}}\right)$ примитивны. Это свойство реализуется только в $\mathfrak{g}^{\#}$-координатах. Таким образом, деформация пространства $V_{\perp}$ как пространстваносителя дополнительного скручивания означает переход к $\mathfrak{g}^{\#-б а з и с у . ~}$

Необходимо указать, что экспоненциальный базис, использованный в этой работе, не может рассматриваться как универсальный. Когда дуальная группа не является разрешимой, должны использоваться другие базисы дуальной группы (например, для максимальной параболической дуальной группы $\mathfrak{G}_{\mathcal{P}}^{\#}$ в линейной группе Ли, содержащей простую подгруппу $\mathfrak{S} \mathfrak{L}(m)$, естественной является алгебра матричных координат).

Благодарности. Автор благодарит П. П. Кулиша за ценные и стимулирующие обсуждения. Работа поддержана РФФИ (грант № 03-01-00593).

\section{Список литературы}

[1] P. Bonneau, M. Gerstenhaber, A. Giaquinto, D. Sternheimer, J. Math. Phys., 45:10 (2004), 3703.

[2] В. Г. Дринфельд, Алгебра и анализ, 1:2 (1989), 30.

[3] М. А. Семенов-Тян-Шанский, ТМФ, 93:2 (1992), 302.

[4] В. Г. Дринфельд, ДАН СССР, 273:3 (1983), 531.

[5] A. I. Ooms, Commun. Alg., 8:1 (1980), 13.

[6] P. P. Kulish, V.D. Lyakhovsky, M. A. del Olmo, J. Phys. A, 32 (1999), 8671.

[7] P. P. Kulish, V.D. Lyakhovsky, J. Phys. A, 33 (2000), L279.

[8] V.D. Lyakhovsky, "Basic twisting factors and the factorization properties of twists", Supersymmetries and Quantum symmetries, Proc. XVII Max Born Symp. (Karpacz, Polland, 21-25 September, 2001), eds. E. Ivanov, S. Krivonos, J. Lukiersky, J. Popovich, World Scientific, Singapore-New Jersey-London-Hong Kong, 2002, 120.

[9] O. V. Ogievetsky, "Hopf structures on the Borel subalgebra of sl(2)", Proc. Winter School of Geometry and Physics (Zdankov, Czech Republic, January, 1993), Ser. II, 37, eds. J. Bures, V. Soucek, Suppl. Rend. Circ. Mat., Circolo Matematico di Palermo, Palermo, 1994, 185.

[10] P. P. Kulish, V. D. Lyakhovsky, A. I. Mudrov, J. Math. Phys., 40 (1999), 4569.

[11] N. Yu. Reshetikhin, Lett. Math. Phys., 20:4 (1990), 331.

[12] Д. Н. Ананикян, П. П. Кулиш, В. Д. Ляховский, Алгебра и анализ, 14:3 (2002), 27.

[13] A. Nijenhuis, R.W. Richardson, Bull. Amer. Math. Soc., 70:3 (1964), 406; 72:1 (1966), 1.

[14] V.D. Lyakhovsky, Czech. J. Phys., 46 (1996), 227.

[15] V.D. Lyakhovsky, M. A. del Olmo, J. Phys. A, 32 (1999), 5343.

[16] V. D. Lyakhovsky, M. E. Samsonov, Journ. Alg. Appl., 1:4 (2002), 413.

Поступила в редакцию 30.10 .2005 , после доработки 24.11.2005 